\title{
Interpreting the Evolving Clumpy Shell Structure of IRC +10216 in Terms of Time Dependent Dust Shell Models
}

\author{
J.M. Winters, T. Blöcker, K.-H. Hofmann, G. Weigelt \\ Max-Planck Institut für Radioastronomie, D-53121 Bonn, Germany
}

The prototypical dust-enshrouded carbon Mira IRC +10216 is known to exhibit intrinsic changes on a time scale of the order of only a few years as revealed, e.g., by CO infrared line profiles (Winters et al. 2000a), its infrared light curves, and by high spatial resolution monitoring in the infrared (Osterbart et al. 2000; Tuthill et al. 2000). In particular, the infrared light curves indicate a possible periodicity on a $\approx 20 \mathrm{yr}$ time scale, i.e. that a recurrent phenomenon might lead to the observed variations. Such multi-periodicity time scales of several $(\approx 10)$ stellar pulsation periods are predicted by consistent hydrodynamical models which include a proper treatment of dust formation (e.g. Winters et al. 2000b). In these models discrete dust layers form in time intervals which are several times longer than the typical pulsation period of an AGB star (Fleischer, Gauger, \& Sedlmayr 1995; Höfner, Feuchtinger, \& Dorfi 1995).

Recent high-spatial resolution monitoring of IRC +10216 in the $\mathrm{K}$ band (Weigelt et al. 1997,1998; Haniff \& Buscher 1998; Osterbart et al. 2000; Tuthill et al. 2000) revealed a fragmented structure of the innermost $\left(\approx 10 R_{*}\right)$ regions of the circumstellar dust shell, which is evolving in relative brightness and spatial separation of the different components on a time scale of $\sim 1 \mathrm{yr}$ (see Weigelt et al., this volume and Fig. 1). However, it is not clear what we are really seeing: thermal emission from dust clouds (Weigelt et al. 1998; Haniff \& Buscher 1998; Tuthill et al. 2000) or direct and scattered stellar light escaping through optically thin regions of the circumstellar shell of overall bipolar structure (Osterbart et al. 2000; Men'shchikov et al. 2001). Due to an instability of the dust formation process caused by the non-local feedback of dust formation on the temperature structure of the dust forming system (see Woitke 2001), one would expect that dust forms in a cloudy structure.

We present an interpretation of the observed morphology in terms of a spherically symmetric, time-dependent dust shell model. The brightest observed component $\mathrm{A}$ is identified with the star while the remaining clumps are conceived as being dust clouds behaving like fragments of the spherical layers formed in the 1D model. The motion of the clumps with respect to $A$ is thereby interpreted as radial expansion of dust clouds blown away from the star by radiation pressure and we follow the time evolution of the intensity emerging from the projected location of the associated dust layers in the model. The respective results of such a model calculation are compared in Fig. 1 with the observed separation (upper r.h.s. panel) and brightness ratio (lower r.h.s. panel) of the brightest component $\mathrm{A}$ and the second brightest component $\mathrm{B}$ seen in the $\mathrm{K}$-band images. The increasing separation as well as the time scale and the intrinsic 'bumpy' 

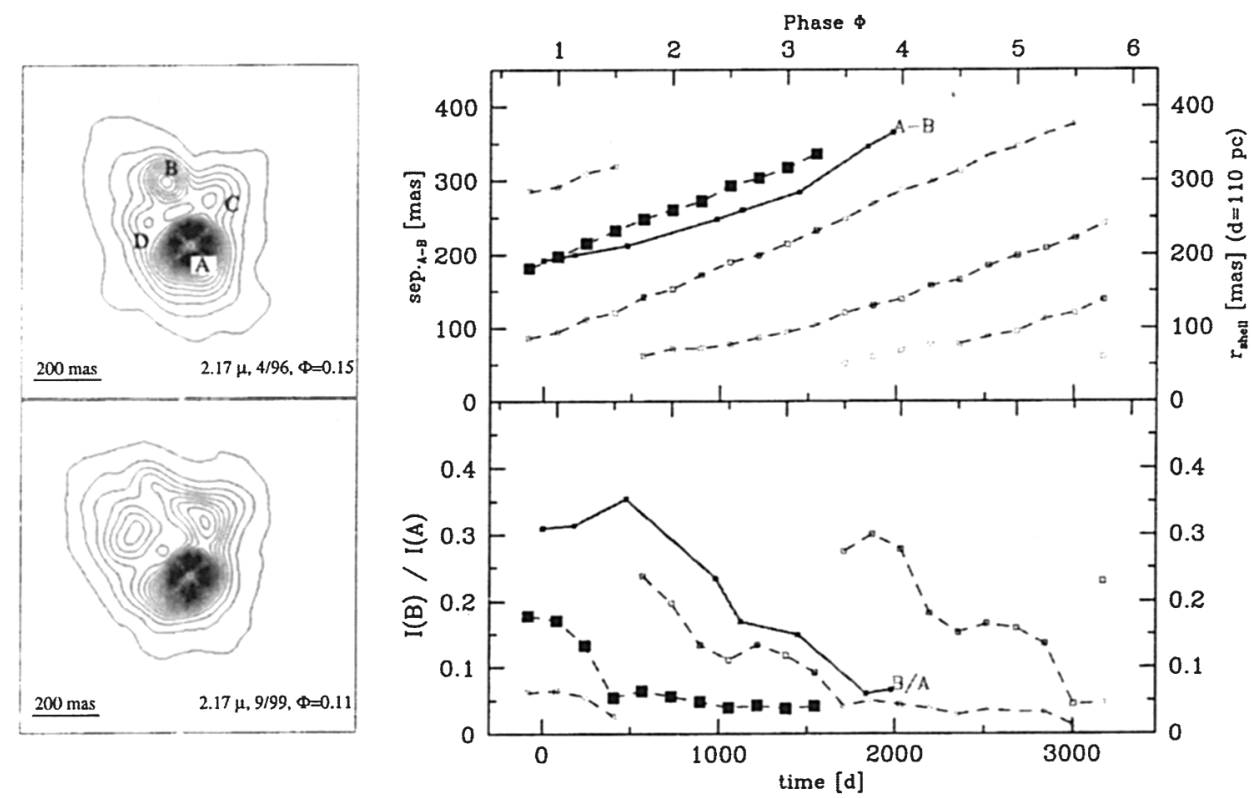

Figure 1. L.h.s. panels: contour maps of IRC +10216 in the $\mathrm{K}$ band in April 1996 (top) and September 1999 (bottom); R.h.s.: Upper panel: observed separation between $A$ and $B$ (solid line) and location of successive dust layers in the model (dashed lines); lower panel: observed (solid) and synthetic (dashed) intensity ratios

structure of the fading of component $\mathrm{B}$ with respect to $\mathrm{A}$ is well reproduced in this scenario. However, the absolute value of the calculated intensity ratio is smaller than observed.

\section{References}

Fleischer A.J., Gauger A., \& Sedlmayr E. 1995, A\&A, 297, 543

Haniff C.A., \& Buscher D.F. 1998 A\&A, 334, L5

Höfner S., Feuchtinger M.U., \& Dorfi E.A. 1995, A\&A, 297, 815

Men'shchikov A.B., Balega Y.Y., Blöcker T., et al. 2001, A\&A, 368, 497

Osterbart R., Balega Y.Y., Blöcker T., et al. 2000, A\&A, 357, 169

Tuthill P., Monnier J., Danchi W., \& Lopez B. 2000, ApJ, 543, 284

Weigelt G., Balega Y., Hofmann K.-H., et al. 1997, in Paresce F. (ed) Science with the VLT Interferometer, Springer, p. 206

Weigelt G., Balega Y., Blöcker T., et al. 1998, A\&A, 333, L51

Winters J.M., Keady J.J., Gauger A., \& Sada P.V. 2000a, A\&A, 359, 651

Winters J.M., Le Bertre T., Jeong K.S., et al. 2000b, A\&A, 361, 641

Woitke P. 2001, Reviews in Modern Astronomy 14, 185 